\title{
Mutimodal Transpose Rotation Mobius Transformation Based Cancellable Template Generation Technique
}

\author{
K.Kanagalakshmi, K.Lakshmipriya
}

\begin{abstract}
Cancelable biometric is such a template security conspire, that replaces a biometric template when the stored template is taken or lost. It is a feature level area transformation where a misshaped variant of a biometric template is produced and coordinated in the transformed space. The issue persevere with the utilization of unique template can be abstained from utilizing cancellable biometrics. In this work, a nonexclusive structure has been intended for producing irreversible portrayal of templates of multimodal which depends on Mobius transformation on unique picture. So the template security is additionally improved. The simulation output of the proposed framework give better execution in Identification of clients. Another strategy called "MTRMT" is proposed to address the issue of stored Templates. The Proposed epic strategy has been assessed with the ongoing fingerprint got from 50 volunteers of veerapandi village in Coimbatore locale. The exploratory outcome shows the better execution of the proposed framework.
\end{abstract} Biometrics.

KEYWORDS: Multimodal, Feature level and Cancelable

\section{INTRODUCTION}

Biometric authentication is the study of building up the identity of a client, towards a framework, in view of his/her physical or conduct properties. Customary arrangements were for the most part unimodal biometric frameworks and may have constrained utilization since no single example from the methodology biometric is adequately exact in certifiable applications, where it is requested from architects to deliver powerful frameworks with low blunder rates and adequate security. By the by, they establish the beginning stage of each examination into the bearing of Multimodal Biometric System (MBS) which looks to diminish a portion of their downsides, by uniting acknowledgment process utilizing numerous templates extracted from a similar individual (e.g., fingerprint, iris, face, hand geometry, stride, keystroke elements).

MBS acknowledge in various degrees of fusion can improve the presentation, hinder ridiculing, increment the general precision of these frameworks, making increasingly solid and most likely, progressively worthy, various related applications[1].

Revised Manuscript Received on December 30, 2019.

* Correspondence Author

Dr.K.Kanagalakshmi*, Principal, Kamalam college of Arts And Science,Anthiyur,Udumalpet,Tamilnadu,India.

kkanagalakshmi@gmail.com.

Mrs.K.Lakshmipriya, Research Scholar, PG \& Research Department of Computer Science, Nehru Arts and Science College, Coimbatore, Tamilnadu, India. abivenkat2007@gmail.com

(c) The Authors. Published by Blue Eyes Intelligence Engineering and Sciences Publication (BEIESP). This is an open access article under the CC BY-NC-ND license (http://creativecommons.org/licenses/by-nc-nd/4.0/)
Cancellable biometrics, additionally alluded to as feature transformations, are structured such that it ought to be computationally hard to recuperate the first data. MBS template security is the source that has spurred various works in the field of the mix of example acknowledgment procedures with the universe of security. From a scholastic research viewpoint, results about the critical preferences in precision, unwavering quality and security of biometric frameworks can guarantee assurance of their stockpiling.

This research was persuaded by extremely late advances in the regions of MBS identication and biometric template assurance. The timeframe for this research is deliberately chosen to serve the exploration in the passage of biometrics in world, mirroring the expanding number of ventures that mean to recommend answers for the security of client's identity, if there should be an occurrence of dangers during on the web exchanges. These reciprocal security advancements can acquire upgrades security and dependability of the frameworks, while fortifying open acknowledgment of the included applications. The principle inspiration driving this decision of fingerprint and iris attributes for a MBS is that fingerprint is the most seasoned and most broadly received biometric innovation and, therefore, is the most experienced of all biometric advances, iris is sealed that it is generally exact and clean biometric innovation among others. Another MBS approach utilizing iris and fingerprint pictures as biometric characteristics has been proposed. The fusion of various features from fingerprint and iris characteristics into a solitary template by utilizing network transpose to rearrange the line and section of every measurement, additionally with the connect work adding the both finger print and iris metric features lastly rotation mobius transform approach is used to make a hybrid cancelable template. As follows Section II Reviews various recent cancelable biometric template generation methods, proposed methodology and experimental study is discussed in Section III, Results and discussion is presented in Section IV and finally Section V concludes the paper.

\section{REVIEW OF LITERATURE}

MBS inquire about endeavors to defeat the drawbacks of Uni-modular biometrics in authentication precision, vigor, and adaptability and gives more extravagant and increasingly dependable biometrics applications. At present, the MBS for the most part centers around the extraction of multimode features at various levels to give a bound together information control interface at the application layer.

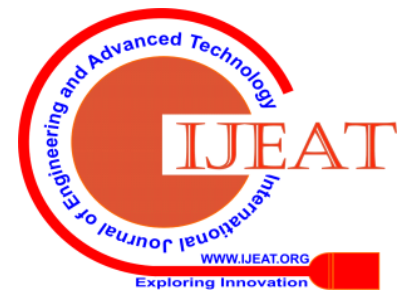


Mehrotra et al. [2] presents multimodal class of characterization for pertinence classifier of vectors, here it consolidated granular and gradual realizing, that deals with huge size of lopsided data's and accomplish efficient execution in MBS grouping and assessment. Abdolahi et al. [3] presented an fusion for multimodal framework utilizing iris and fingerprint utilizying fuzzy logic and the conspicuous improvement in acknowledgment rate was accomplished. Moreover hence, the technique doesn't provides calculated examination in adequacy of the fusion procedure, thus acquired impact was ineffectively summed up. Miao et al. [4] presents an system of receptacle dependent classifier technique for multibiometrics fusion, here it insert coordinating scores into another picture pixel space and acquire more extravagant feature data while executing picture based biometrics. Chen et al. [5] presents an system for fingerprint and face pictures fusion utilizing a kind of center layer semantic features extracted from neighborhood feature-picture grid. Be that as it may, it is as yet uncertain whether this feature has great feature articulation for a wide range of biometrics.

Kanade et al. [6] consolidated two variables, iris and secret phrase, to create cancelable iris templates. In particular, a client explicit key is utilized to shue the iris code and an Error Correcting Code (ECC) is utilized to diminish feature variety to accomplish better acknowledgment execution. Pillai et al. [7] Structured cancelable iris biometrics dependent on sectored arbitrary projections. Two stages, feature extraction and irregular projections, are remembered for this technique. The trial results show that the rule for cancelability is met.

K. Kanagalakshmi [8] proposed a technique called Reciprocated Magnitude and Complex Conjugate-Phase transformation. It is a cancelable and unavoidable biometric template creating procedure. It is evaluated in various certainties like Cancelability, Irrevocability and Security. K.Kanagalakshmi and E.Chandra [9], "Novel Complex Conjugate-Phase Transform strategy for cancelable and unavoidable biometric template for fingerprints, the complex conjugate stage Transform are utilized to ascertain normal time of biometric template creation and coordinating are likewise determined. Most extreme memory utilization of the biometric template is likewise determined.

\section{PROPOSED METHOD: MULTIMODAL TRANSPOSE ROTATION MOBIUS TRANSFORMATION TECHNIQUE (MTRMT)}

MBS is termed to be a best authentication because of the blend of novel qualities of a person. In this proposed framework iris and fingerprint is consolidated and utilized as an attribute of recognizing an individual. Here cancelable multimodal biometric picture template is created to verify the recognzation of an individual. In this system it involves 6 phases. They are data gathering, preprocessing of image, extraction of minutiae, post processing, applying proposed technique and creation of cancelable template as shown in Figure 1.The raw data of finger print and iris are given as information and afterward the progression astute methodology of preprocessing is pursued to expel the commotion from the given image data by utilizing filter called Gabor. The Region of Interest (ROI) is acquired and the features are extracted independently. The transpose of matrix then summation work is applied lastly Rotation mobius transformation is inferred to make a hybrid cancellable template feature sets for all information that are given as input. These qualities are stored in database. The framework stream chart of proposed strategy is indicated in Figure.1.

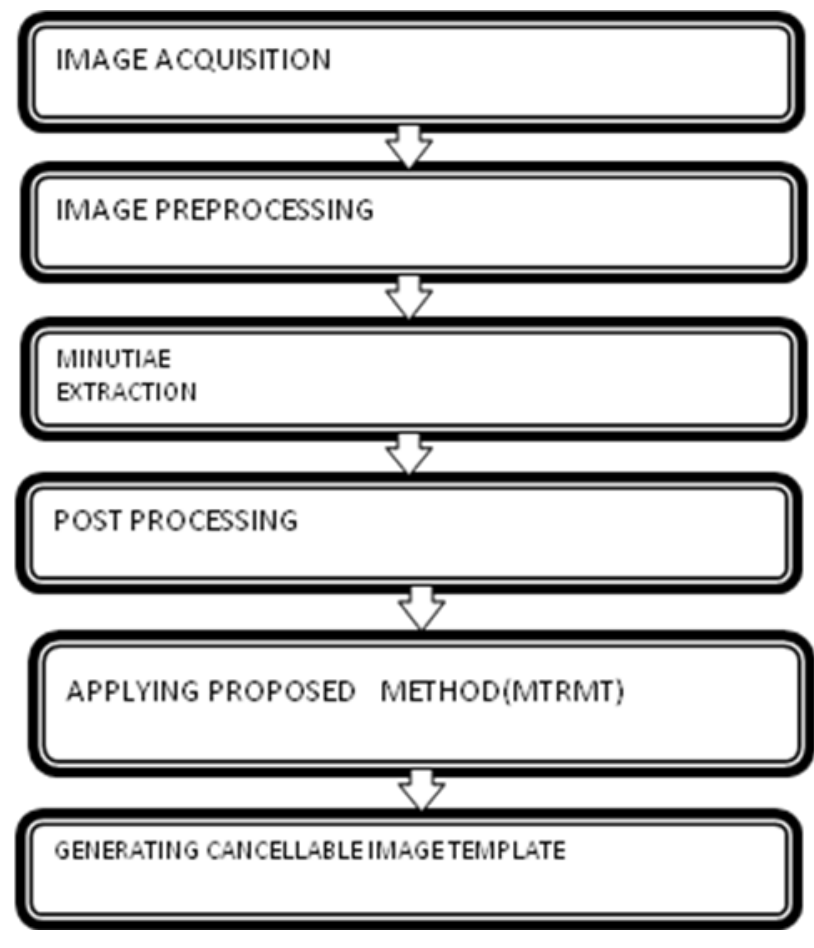

Figure 1: System Flow Diagram of Proposed Technique

\subsection{Objective Of Cancelable Biometric Template}

\section{Generation}

The indispensable goal of the proposed technique is to create a hybrid cancelable template. This creation of template is conceivable by moving the area or applying same strategy on feature point to change the estimation of particulars position.

\section{Detailed Procedure:}

Stage 1: Input the raw pictures of finger print and iris.

Stage 2: Remove the commotion in unique picture utilizing Gabor filter and choose Region of Interest[8].

Stage 3: Select the Feature of Interest and the procedure for thinning is finished.

Stage 4: Transpose the estimation of fingerprint (A) as $A^{T}$ and Iris (B) as $\mathrm{B}^{\mathrm{T}}$.

Stage 5: Concatenation of Matrix is applied $A^{T}+B^{T}$.

Stage 6: Rotation Mobius Transformation is actualized[10].

Stage 7: Generate the output of cancelable template design.

The stream chart of Generating cancelable template utilizing MTRMT is appeared in Figure 2 


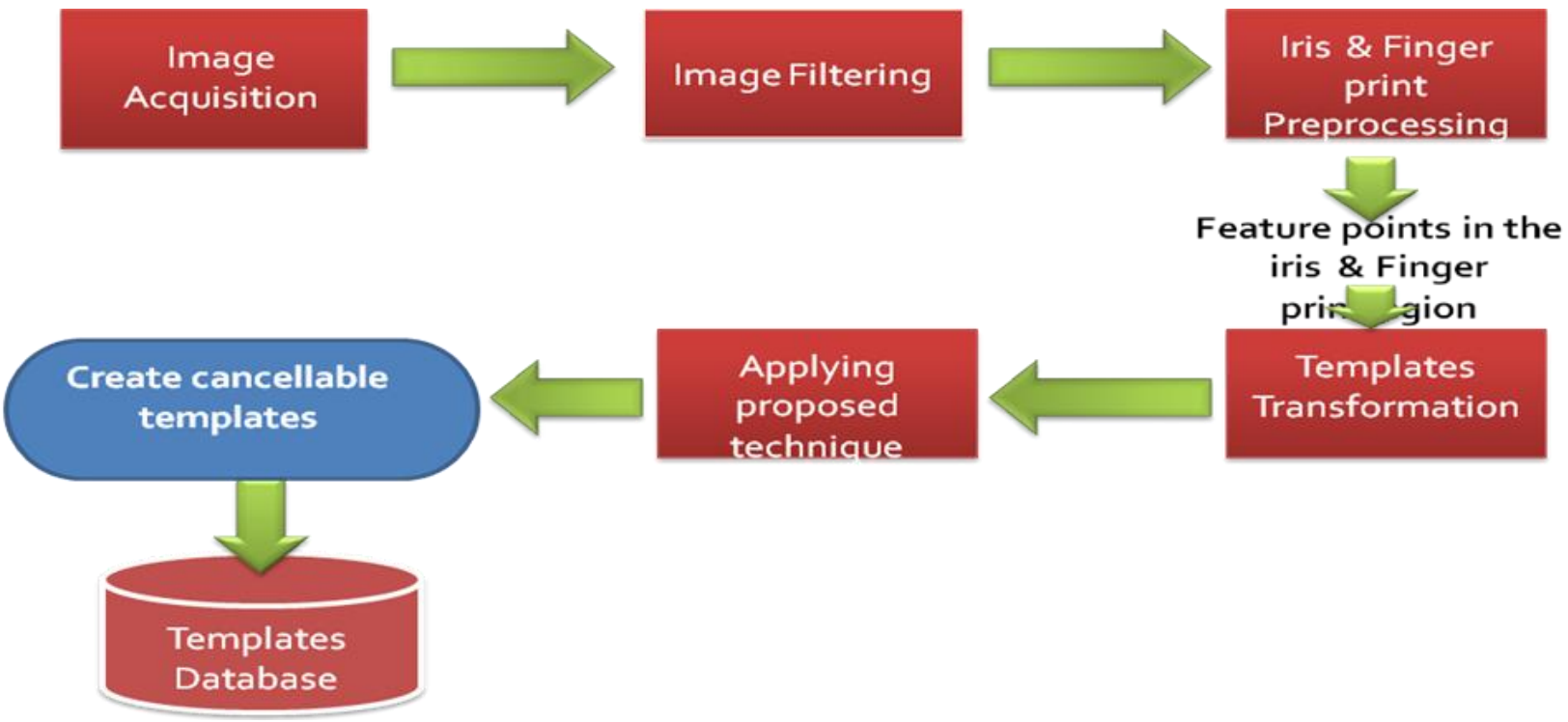

Figure 2: Flow diagram of MTRMT

By following the above stages 1,2 and 3 the different feature set of Iris and Finger print are acquired to make the biometric as hybrid one. Stage 4, 5 and 6 assume significant job in the proposed work. The extracted features are stored in a different matrix of finger print (A) and Iris (B).

In proposed strategy, 2 primary goals are pursued, they are

1. The feature of interest for unique picture is transposed by inferring the matrix transpose technique. and iris.

Here this method is applied for both finger print

In Stage 4, the numerical transpose of matrix function is utilized to get $\mathrm{A}^{\mathrm{T}}$ and $\mathrm{B}^{\mathrm{T}}$ individually.

Where $\mathrm{A}$ and $\mathrm{B}$ are two matrices of same order and $\mathrm{T}$ indicates to transpose of matrix[10].

The transpose of matrix resembles

$\mathrm{A}=[\mathrm{aij}]_{\mathrm{m} \times \mathrm{n}}$ and $\mathrm{B}=[\mathrm{aij}]_{\mathrm{m} \times \mathrm{n}}$

then its transpose which is written as

$\mathrm{A}^{\mathrm{T}}=[\mathrm{aji}]_{\mathrm{n} \times \mathrm{m}}$ and $\mathrm{B}^{\mathrm{T}}=[\mathrm{aji}]_{\mathrm{n} \times \mathrm{m}}$

On the off chance that transpose of any matrix is inferred, at that point request of matrix gets turned around. Component present at $(\mathrm{i}, \mathrm{j})$ th position in a given matrix $\mathrm{A}$ and $\mathrm{B}$ goes to $(\mathrm{j}, \mathrm{i})$ th position in transpose of matrix $\mathrm{A}$ and $\mathrm{B}$.

2. At that point the output of transposed finger print and iris is added to roll out certain improvements in area of feature. This delivers some cancelablity in features.

In Stage 5, concatenation of two individual transpose matrix $\mathrm{A}^{\mathrm{T}}$ and $\mathrm{B}^{\mathrm{T}}$ is finished by utilizing Matrix expansion, the way toward consolidating the output of transposed feature by both iris and finger print. Here, feature of iris and finger print are joined was appeared in following condition.

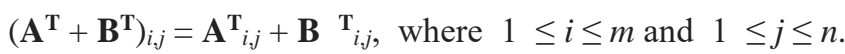

The estimation of result from Stage 5 is passed to Rotation mobius transform to frame the cancelable hybrid templates.

\subsection{Transpose Rotation Mobius Transformation}

The spot of creating cancelable template is in the Stage 6 . Here Rotation mobius transformation is utilized for making hybrid template.

$$
f(z)=\frac{a z+b}{c z+d}
$$

Where $\mathrm{z}$ is intricate variable, the coefficients $\mathrm{a}, \mathrm{b}, \mathrm{c}, \mathrm{d}$ are values of complex which fulfilling $a d-b c \neq 0$.

$$
\mathrm{R}^{\mathrm{T}}=R^{-1}, R^{T} R=I
$$

A general Mobius Transformation related to the unit circle in $\mathrm{R}$, in which the plane $\mathrm{x}=0$ and guide it to the unit circle by opposite stereographic projection from the North Pole[10]. Finishing the identification by mapping the point at interminability toward the North Pole, the Möbius transformations compare to the holomorphic automorphisms of this Riemann circle. Be that as it may, it isn't evident what the holomorphic automorphisms of the circle resemble and it requires some exertion and refinement to get an unmistakable image of the Mobius transformations along these lines.

In this article the shape joined with this new feature is exceptionally oppressive for recognizing genuine clients by Rotation mobius transform calculation ventures as pursues:

i. Tile the matrixes by non covering range squares (for example $8 * 8$ ) and bigger (for example $16 * 16$ ), perhaps covering space blocks. 


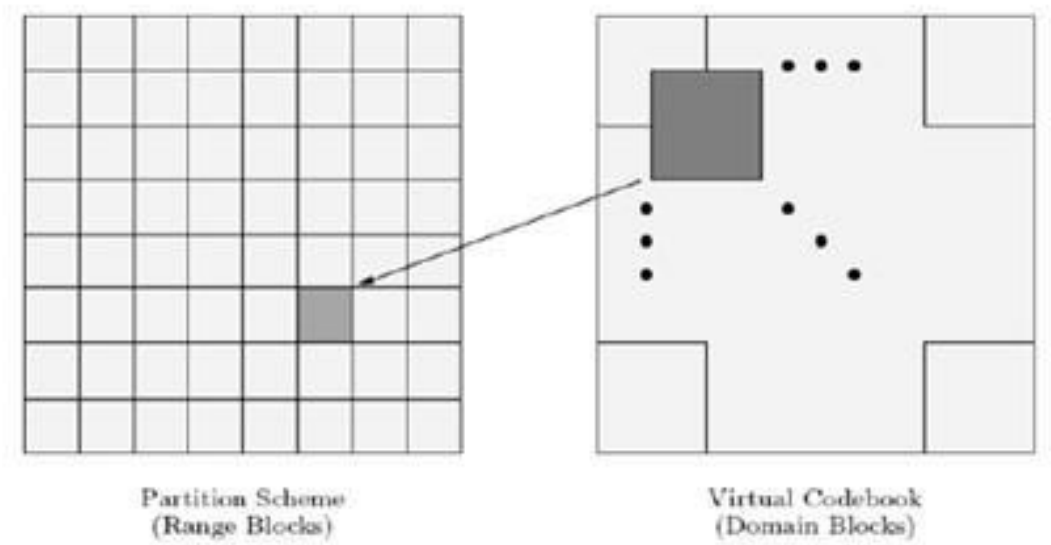

Figure 3: Domain \& Range blocks in PIFS coding.

ii. A set of acceptable square transforms is characterized, comprising of a withdrawal of the square help by a factor of two on each side by averaging neighboring pixels.

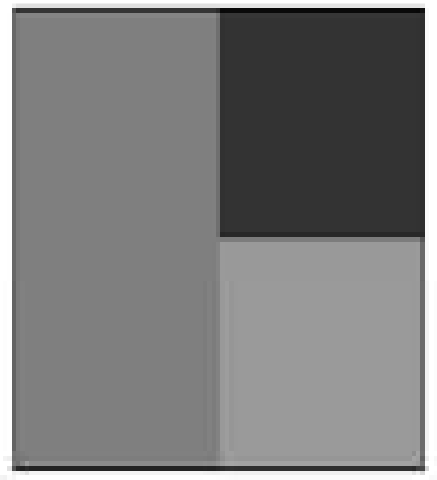

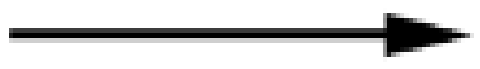

\section{Spatial contraction}

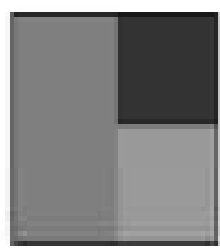

\section{Contracted domain block}

\section{Domain block}

Figure 4: Spatial contraction of a Domain block

iii. Process is pursued as in Fig(5)(6) by the use of one of the eight pivots and reflections making up the isometrics of a square[10].
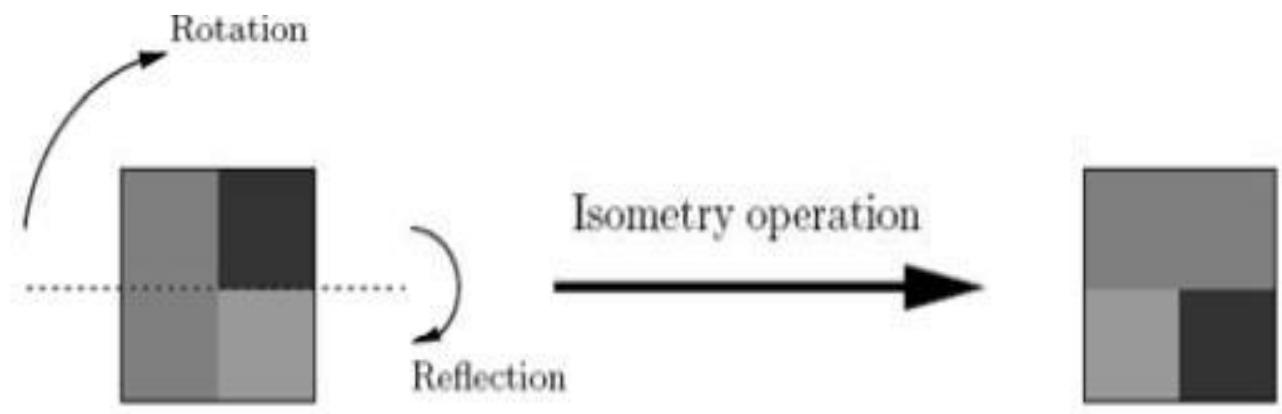

Figure 5: An isometric applied to domain block. 

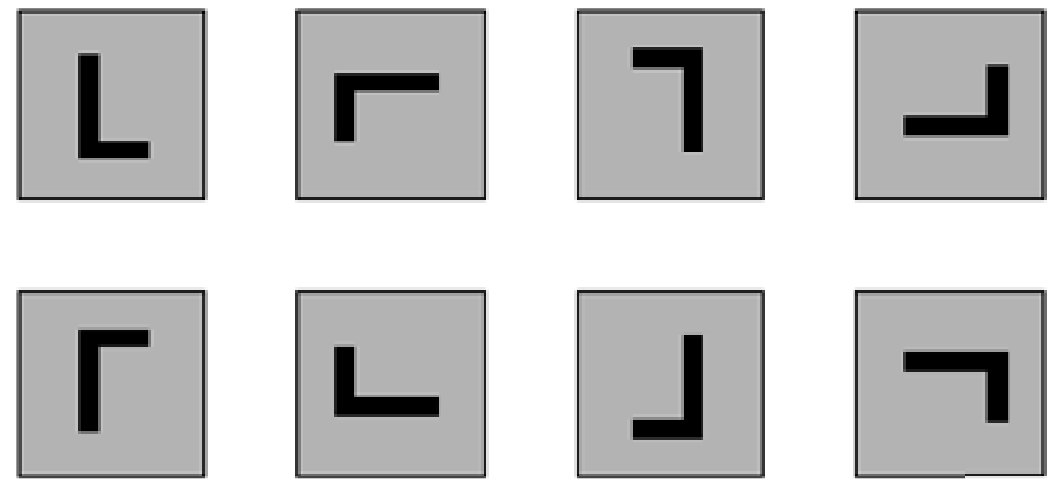

Figure 6: The Square isometries.

iv. Finally a relative transform on the pixel powers of the range square (contracted Domain square) is applied(Figure.7).

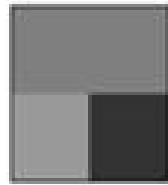

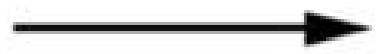

Intensity scaling
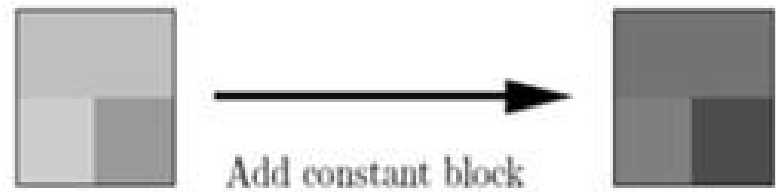

\section{Rotated and contracted domain block}

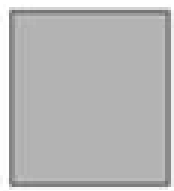

Figure 7: An Affine transformation applied to the domain block.

v. The Enrollment Phase comprises of finding for each range obstruct a space hinder for which the pixel esteems can be $p$ made near those of the range hinder by the utilization of an acceptable transform.

vi. Once Enrollment is finished, the picture is spoken to by a rundown containing the chose area square and transform parameters for each range square [10].

vii. The picture is decoded by iteratively transforming a discretionary starting picture utilizing the transform comprising of the association of the transforms for each range square.

viii. Finally the hybrid templates feature sets are stored in the database.

\section{EXPERMENTAL RESULTS AND DISCUSSIONS:}

The consequences of the proposed framework are examined with different stages of execution. Each stage of the proposed technique must demonstrate the advancement of utilizing this framework in the spot of existing framework. There are three stages of result in the proposed framework; they are Enrollment stage, Preprocessing stage and Verification stage. The brief work is appeared underneath in stage by stage.

\subsection{Enrollement Stage}

The raw image data is given to the proposed framework by GUI based strategy. The enrollement of finger print and iris are made. This stage is othewise called as enrollement phase. The enrollement of client is appeared in Figure 8.

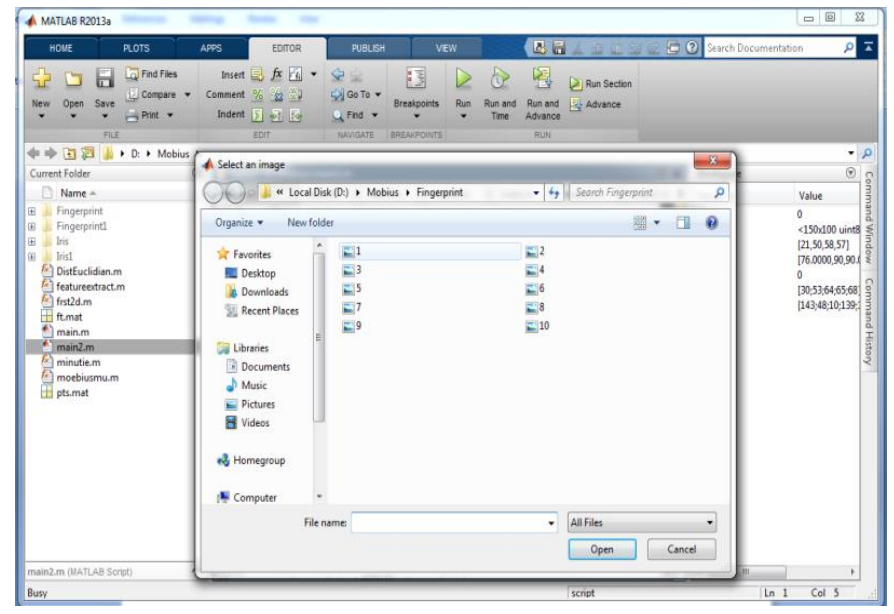

Figure 8: Enrollment stage

\subsection{Preprocessing stage}

Preprocessing steps of the biometric framework is pursued to evacuate the commotion like Gaussian, salt and pepper and so forth. The cropping, thinning minutiae extraction process are made to prepare the picture to infer proposed framework. The Preprocessing stage is demonstrated in Figure [9],[10],[11]. 


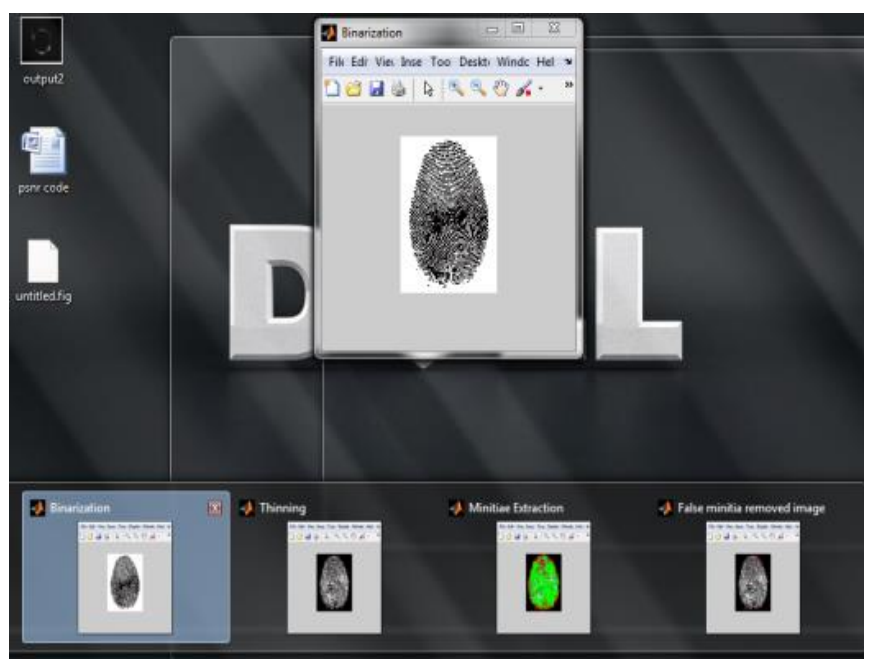

Figure 9: Binarization

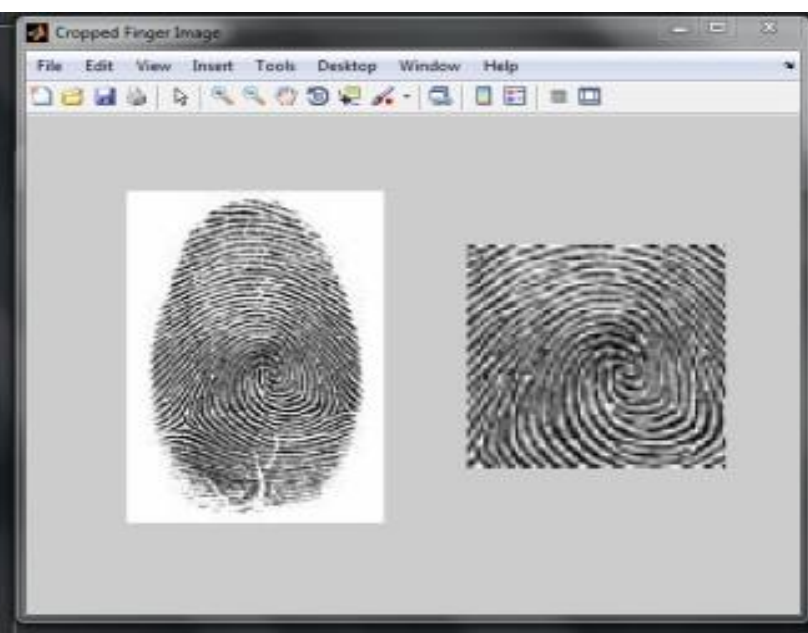

Figure 10: Cropped fingerprint

\begin{tabular}{|c|c|c|c|c|c|c|c|c|c|c|}
\hline \multirow[t]{2}{*}{ Image } & \multirow[t]{2}{*}{ Trait 1} & \multicolumn{2}{|c|}{ Gaussian filter } & \multicolumn{2}{|c|}{ Gabor filter } & \multirow[t]{2}{*}{ Trait 2} & \multicolumn{2}{|c|}{ Gaussian filter } & \multicolumn{2}{|c|}{ Gabor filter } \\
\hline & & PSNR & MSE & PSNR & MSE & & PSNR & MSE & PSNR & MSE \\
\hline 1 & \multirow{10}{*}{ Iris } & 30.7985 & 6.6055 & 27.876 & 9.2478 & \multirow{10}{*}{ FP } & 26.4049 & 12.1981 & 23.4824 & 17.0774 \\
\hline 2 & & 31.1324 & 7.0781 & 28.2099 & 9.9094 & & 35.687 & 3.779 & 32.7645 & 5.2906 \\
\hline 3 & & 30.2595 & 7.8265 & 27.3369 & 10.9571 & & 28.8106 & 8.7757 & 25.8881 & 12.286 \\
\hline 4 & & 31.2196 & 7.0075 & 28.297 & 9.8104 & & 32.3788 & 5.9396 & 29.4562 & 8.3155 \\
\hline 5 & & 30.4976 & 7.6149 & 27.575 & 10.6608 & & 32.4504 & 5.7239 & 29.5278 & 8.0135 \\
\hline 6 & & 19.9172 & 23.1194 & 23.439 & 15.4129 & & 15.5236 & 42.6935 & 19.0454 & 28.4623 \\
\hline 7 & & 20.2511 & 24.7734 & 23.7729 & 16.5156 & & 24.8057 & 13.2265 & 28.3275 & 8.8177 \\
\hline 8 & & 19.3781 & 27.3927 & 22.8999 & 18.2618 & & 17.9293 & 30.7151 & 21.4511 & 20.4767 \\
\hline 9 & & 20.3382 & 24.5261 & 23.8601 & 16.3507 & & 21.4974 & 20.7886 & 25.0192 & 13.8591 \\
\hline 10 & & 19.6162 & 26.652 & 23.138 & 17.768 & & 21.569 & 20.0337 & 25.0908 & 13.3558 \\
\hline
\end{tabular}

Table :1 PSNR \& MSE Ratio of image using Gaussian and Gabor filter

\subsection{Verification : True result}

Approval is the significant errand done by the framework to recognize the certifiable client. This procedure is finished by contrasting the result with enrollment of stored value. Here stored esteem is capacity of every single trained image template and it is template made during

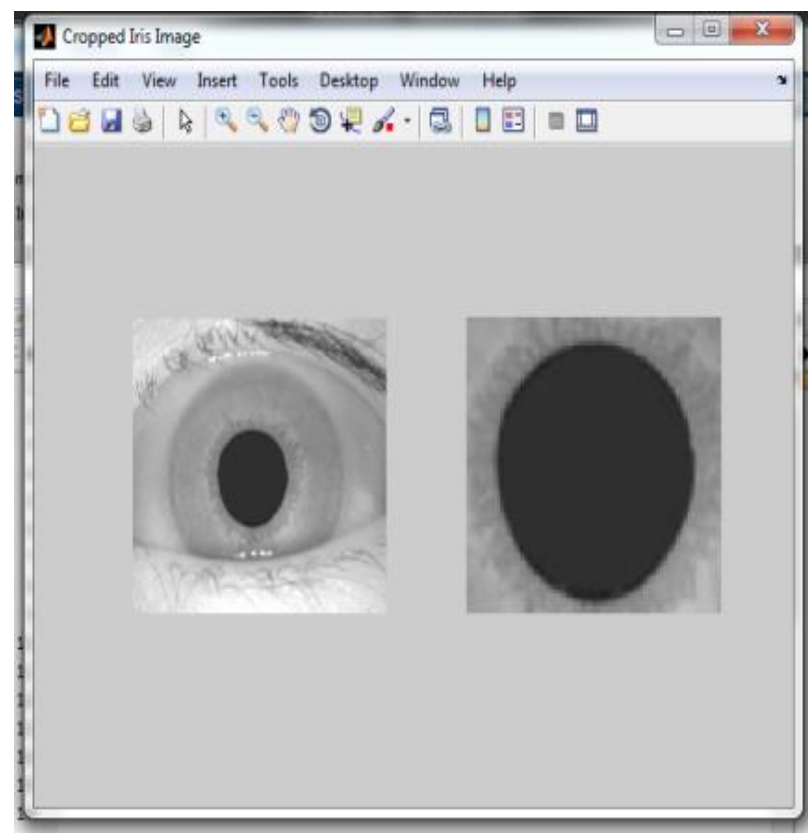

Figure 11: Cropped iris

The standard filter Gabor is utilized to evacuate the clamors. The commotion proportion of the given images is outfitted utilizing filters Gaussian and Gabor are appeared in Table 1. enrollement process. If stored esteem contains the estimation of selected worth the client authentication is sucessful, if not the client get to is deneid. The correlation template estimation of stored values and values of enrolled are appeared in the figure 12 .

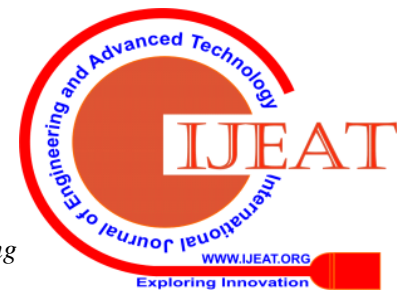


Stored value

\begin{tabular}{|c|c|c|c|c|c|c|}
\hline & 1 & 2 & 3 & 4 & 5 & 6 \\
\hline 1 & $3.8334 e-07$ & $1.8231 \mathrm{e}-07$ & $1.7568 \mathrm{e}-07$ & $1.9437 \mathrm{e}-07$ & $1.6919 \mathrm{e}-07$ & $1.2700 \mathrm{e}-07$ \\
\hline 2 & $1.6200 \mathrm{e}-07$ & $1.3742 \mathrm{e}-07$ & $2.5146 \mathrm{e}-07$ & $3.3446 e-07$ & $2.2301 \mathrm{e}-07$ & $1.1416 \mathrm{e}-07$ \\
\hline 3 & $1.3463 \mathrm{e}-07$ & $2.5723 \mathrm{e}-07$ & $5.6330 \mathrm{e}-07$ & $6.5275 e-07$ & $3.6742 e-07$ & $1.6735 \mathrm{e}-07$ \\
\hline 4 & $1.7287 e-07$ & $5.5473 \mathrm{e}-07$ & $1.2996 \mathrm{e}-06$ & $1.3063 \mathrm{e}-06$ & $5.7290 \mathrm{e}-07$ & $2.0883 \mathrm{e}-07$ \\
\hline 5 & $2.0517 e-07$ & $6.0959 e-07$ & $1.3743 e-06$ & $1.3151 \mathrm{e}-06$ & $5.2177 e-07$ & $1.7029 \mathrm{e}-07$ \\
\hline 6 & $2.1153 e-07$ & $3.1350 \mathrm{e}-07$ & $5.4290 \mathrm{e}-07$ & $4.9302 \mathrm{e}-07$ & $2.0187 e-07$ & $8.6292 \mathrm{e}-08$ \\
\hline 7 & $2.4578 \mathrm{e}-07$ & $1.2629 \mathrm{e}-07$ & $9.6127 e-08$ & $7.3107 e-08$ & $3.7471 \mathrm{e}-08$ & $3.4802 \mathrm{e}-08$ \\
\hline 8 & $1.9644 \mathrm{e}-07$ & $6.0051 \mathrm{e}-08$ & $1.4209 \mathrm{e}-08$ & $5.5869 \mathrm{e}-09$ & $4.7299 \mathrm{e}-09$ & $1.2226 \mathrm{e}-08$ \\
\hline 9 & $8.6818 \mathrm{e}-08$ & $3.5923 \mathrm{e}-08$ & $9.2958 \mathrm{e}-09$ & $2.4292 \mathrm{e}-09$ & $4.2120 \mathrm{e}-09$ & $1.7238 \mathrm{e}-08$ \\
\hline & $3.9739 \mathrm{e}-08$ & $3.4767 e-08$ & $2.0573 e-08$ & $1.5041 \mathrm{e}-08$ & $3.8395 e-08$ & $1.0278 \mathrm{e}-07$ \\
\hline
\end{tabular}

Enrolled value

\begin{tabular}{|c|c|c|c|c|c|c|}
\hline \multirow{2}{*}{\multicolumn{7}{|c|}{$\begin{array}{l}\mathrm{ft} \times \mathrm{ft}\{1,1\} \times \mathrm{f} \\
\boxplus \mathrm{fts}<75 \times 75 \text { double }>\end{array}$}} \\
\hline & & & & & & \\
\hline & 1 & 2 & 3 & 4 & 5 & 6 \\
\hline 1[ & $3.8334 \mathrm{e}-07$ & $1.8231 \mathrm{e}-07$ & $1.7568 \mathrm{e}-07$ & $1.9437 \mathrm{e}-07$ & $1.6919 \mathrm{e}-07$ & $1.2700 \mathrm{e}-07$ \\
\hline 2 & $1.6200 \mathrm{e}-07$ & $1.3742 \mathrm{e}-07$ & $2.5146 \mathrm{e}-07$ & $3.3446 \mathrm{e}-07$ & $2.2301 \mathrm{e}-07$ & $1.1416 \mathrm{e}-07$ \\
\hline 3 & $1.3463 \mathrm{e}-07$ & $2.5723 \mathrm{e}-07$ & $5.6330 \mathrm{e}-07$ & $6.5275 e-07$ & $3.6742 \mathrm{e}-07$ & $1.6735 \mathrm{e}-07$ \\
\hline 4 & $1.7287 \mathrm{e}-07$ & $5.5473 \mathrm{e}-07$ & $1.2996 \mathrm{e}-06$ & $1.3063 \mathrm{e}-06$ & $5.7290 \mathrm{e}-07$ & $2.0883 \mathrm{e}-07$ \\
\hline 5 & $2.0517 \mathrm{e}-07$ & $6.0959 \mathrm{e}-07$ & $1.3743 \mathrm{e}-06$ & $1.3151 \mathrm{e}-06$ & $5.2177 e-07$ & $1.7029 \mathrm{e}-07$ \\
\hline 6 & $2.1153 \mathrm{e}-07$ & $3.1350 \mathrm{e}-07$ & $5.4290 \mathrm{e}-07$ & $4.9302 \mathrm{e}-07$ & $2.0187 e-07$ & $8.6292 e-08$ \\
\hline 7 & $2.4578 \mathrm{e}-07$ & $1.2629 \mathrm{e}-07$ & $9.6127 e-08$ & $7.3107 e-08$ & $3.7471 \mathrm{e}-08$ & $3.4802 \mathrm{e}-08$ \\
\hline 8 & $1.9644 \mathrm{e}-07$ & $6.0051 \mathrm{e}-08$ & $1.4209 \mathrm{e}-08$ & $5.5869 \mathrm{e}-09$ & $4.7299 \mathrm{e}-09$ & $1.2226 \mathrm{e}-08$ \\
\hline 9 & $8.6818 \mathrm{e}-08$ & $3.5923 \mathrm{e}-08$ & $9.2958 \mathrm{e}-09$ & $2.4292 \mathrm{e}-09$ & $4.2120 \mathrm{e}-09$ & $1.7238 \mathrm{e}-08$ \\
\hline 10 & $3.9739 \mathrm{e}-08$ & $3.4767 \mathrm{e}-08$ & $2.0573 \mathrm{e}-08$ & $1.5041 \mathrm{e}-08$ & $3.8395 \mathrm{e}-08$ & $1.0278 \mathrm{e}-07$ \\
\hline
\end{tabular}

Figure 12: Match success Template value

Here the result in template of enrolled is same as stored template value, in this way the client is certified and allowed to acccess. The True verification is indicated the figure 13.

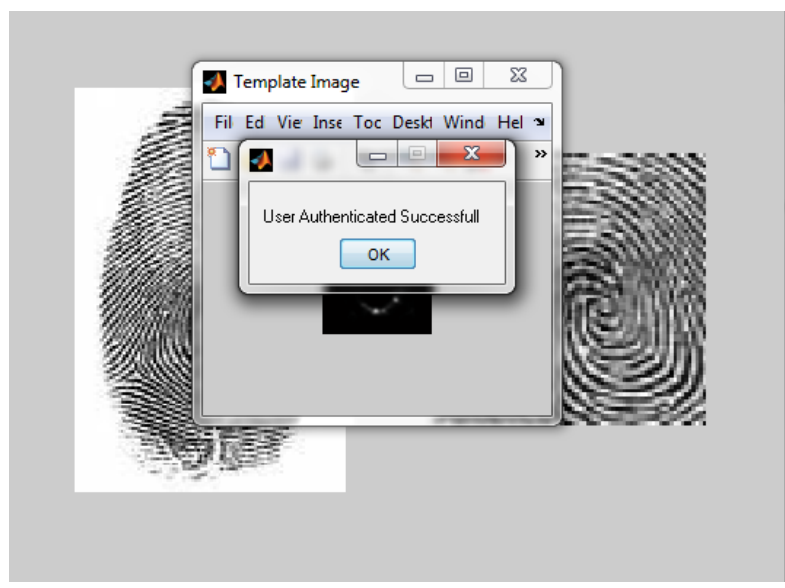

Figure 13: True Verification

\subsection{Verification : False result}

Here the template of stored value doesn't match the estimation of enrolled template. The result is appeared in figure 14 .

\section{Stored value}

\begin{tabular}{|c|c|c|c|c|c|c|}
\hline \multicolumn{7}{|c|}{$\mathrm{ft} \times \mathrm{ft}\{1,1\} \times$} \\
\hline \multicolumn{7}{|c|}{$\boxplus \mathrm{ft}\{1,1\}<75 \times 75$ double $>$} \\
\hline & 1 & 2 & 3 & 4 & 5 & 6 \\
\hline 1 & $3.8334 \mathrm{e}-07$ & $1.8231 \mathrm{e}-07$ & $1.7568 \mathrm{e}-07$ & $1.9437 \mathrm{e}-07$ & $1.6919 \mathrm{e}-07$ & $1.2700 \mathrm{e}-07$ \\
\hline 2 & $1.6200 \mathrm{e}-07$ & $1.3742 \mathrm{e}-07$ & $2.5146 \mathrm{e}-07$ & $3.3446 \mathrm{e}-07$ & $2.2301 \mathrm{e}-07$ & $1.1416 \mathrm{e}-07$ \\
\hline 3 & $1.3463 \mathrm{e}-07$ & $2.5723 \mathrm{e}-07$ & $5.6330 \mathrm{e}-07$ & $6.5275 \mathrm{e}-07$ & $3.6742 \mathrm{e}-07$ & $1.6735 \mathrm{e}-07$ \\
\hline 4 & $1.7287 e-07$ & $5.5473 \mathrm{e}-07$ & $1.2996 \mathrm{e}-06$ & $1.3063 \mathrm{e}-06$ & $5.7290 \mathrm{e}-07$ & $2.0883 e-07$ \\
\hline 5 & $2.0517 e-07$ & $6.0959 \mathrm{e}-07$ & $1.3743 \mathrm{e}-06$ & $1.3151 \mathrm{e}-06$ & $5.2177 e-07$ & $1.7029 \mathrm{e}-07$ \\
\hline 6 & $2.1153 e-07$ & $3.1350 \mathrm{e}-07$ & $5.4290 \mathrm{e}-07$ & $4.9302 \mathrm{e}-07$ & $2.0187 e-07$ & $8.6292 \mathrm{e}-08$ \\
\hline 7 & $2.4578 \mathrm{e}-07$ & $1.2629 \mathrm{e}-07$ & $9.6127 e-08$ & $7.3107 e-08$ & $3.7471 \mathrm{e}-08$ & $3.4802 \mathrm{e}-08$ \\
\hline 8 & $1.9644 \mathrm{e}-07$ & $6.0051 \mathrm{e}-08$ & $1.4209 \mathrm{e}-08$ & $5.5869 \mathrm{e}-09$ & $4.7299 \mathrm{e}-09$ & $1.2226 \mathrm{e}-08$ \\
\hline 9 & $8.6818 \mathrm{e}-08$ & $3.5923 \mathrm{e}-08$ & $9.2958 \mathrm{e}-09$ & $2.4292 \mathrm{e}-09$ & $4.2120 \mathrm{e}-09$ & $1.7238 \mathrm{e}-08$ \\
\hline 10 & $3.9739 \mathrm{e}-08$ & $3.4767 e-08$ & $2.0573 \mathrm{e}-08$ & $1.5041 \mathrm{e}-08$ & $3.8395 e-08$ & $1.0278 \mathrm{e}-07$ \\
\hline
\end{tabular}

Enrolled value

\begin{tabular}{|c|c|c|c|c|c|c|}
\hline \multicolumn{7}{|c|}{$\mathrm{fts} \times$} \\
\hline \multicolumn{7}{|c|}{$\boxplus$ fts $<75 \times 75$ double $>$} \\
\hline & 1 & 2 & 3 & 4 & 5 & 6 \\
\hline 1 & $2.5589 \mathrm{e}-07$ & $1.5837 \mathrm{e}-07$ & $1.2060 \mathrm{e}-07$ & $8.0320 \mathrm{e}-08$ & $9.6926 \mathrm{e}-08$ & $1.3747 \mathrm{e}-07$ \\
\hline 2 & $3.7679 \mathrm{e}-07$ & $1.6987 e-07$ & $8.9676 \mathrm{e}-08$ & $4.1787 e-08$ & $4.0939 \mathrm{e}-08$ & $6.1745 \mathrm{e}-08$ \\
\hline 3 & $5.2645 e-07$ & $2.5193 \mathrm{e}-07$ & $1.0139 \mathrm{e}-07$ & $3.1149 \mathrm{e}-08$ & $1.7709 \mathrm{e}-08$ & $1.9757 e-08$ \\
\hline 4 & $6.9942 \mathrm{e}-07$ & $4.4742 \mathrm{e}-07$ & $1.6047 \mathrm{e}-07$ & $3.8212 \mathrm{e}-08$ & $1.3782 \mathrm{e}-08$ & $6.8174 \mathrm{e}-09$ \\
\hline 5 & $8.3604 \mathrm{e}-07$ & $5.3025 \mathrm{e}-07$ & $1.6792 \mathrm{e}-07$ & $3.0829 \mathrm{e}-08$ & $9.7690 \mathrm{e}-09$ & $3.7282 \mathrm{e}-09$ \\
\hline 6 & $8.7502 e-07$ & $3.8532 \mathrm{e}-07$ & $1.1066 \mathrm{e}-07$ & $1.6393 \mathrm{e}-08$ & $4.6868 \mathrm{e}-09$ & $2.8052 \mathrm{e}-09$ \\
\hline 7 & $1.0959 \mathrm{e}-06$ & $3.0441 \mathrm{e}-07$ & $8.0962 \mathrm{e}-08$ & $1.4998 \mathrm{e}-08$ & $2.4502 \mathrm{e}-09$ & $1.8109 \mathrm{e}-09$ \\
\hline 8 & $2.0118 \mathrm{e}-06$ & $5.5516 \mathrm{e}-07$ & $1.0890 \mathrm{e}-07$ & $2.0228 \mathrm{e}-08$ & $2.6757 e-09$ & $1.1518 \mathrm{e}-09$ \\
\hline 9 & $3.7280 \mathrm{e}-06$ & $1.3755 \mathrm{e}-06$ & $3.4044 \mathrm{e}-07$ & $6.9745 e-08$ & $8.2382 \mathrm{e}-09$ & $1.6056 \mathrm{e}-09$ \\
\hline 10 & $4.0477 e-06$ & $2.2980 \mathrm{e}-06$ & $9.2686 \mathrm{e}-07$ & $2.5623 \mathrm{e}-07$ & $3.4833 \mathrm{e}-08$ & $1.1065 \mathrm{e}-08$ \\
\hline
\end{tabular}

Figure 14: Verification denied Template value

Any qualities utilized in this proposed framework i.e, if iris and finger print get mismatch ,the result in stored data won't contain the estimation of enrolled data then the client is preclude utilize the framework. The False match status are appeared in figure 15.

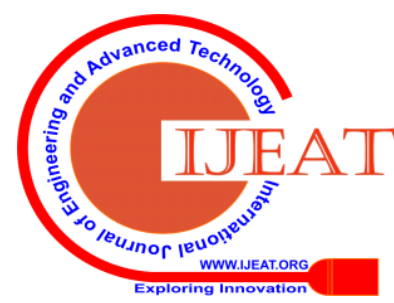




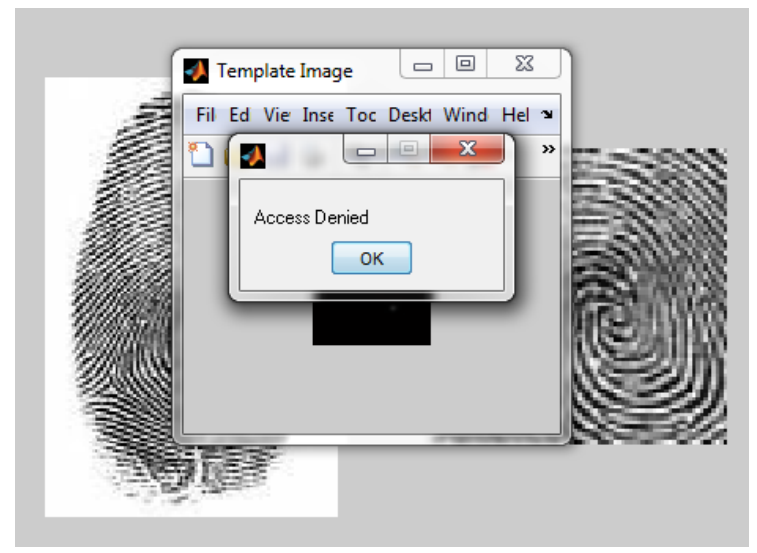

Figure 15: False Verification

The results of the proposed framework is estimated as far as execution time and memory portion. After verification it is important to check the memory size and time devoured of the value obtained. These 2 parameters assumes imperative job in ascertaining the nature of the proposed strategy. The tables of the referenced parameters are appeared to improve the quality verification of the framework to demonstrate the proposed strategy as better. In following Table 2 shows, the Memory size, Table 3 shows the average execution time stage by stage and Table 4 shows the performance.

Table :2 Memory Size

\begin{tabular}{|c|c|c|c|c|c|}
\hline Image & $\begin{array}{c}\text { FP in } \\
\text { Kb }\end{array}$ & $\begin{array}{c}\text { Iris in } \\
\text { Kb }\end{array}$ & $\begin{array}{c}\text { ROI of FP } \\
\text { in Kb }\end{array}$ & $\begin{array}{c}\text { ROI of Iris } \\
\text { in Kb }\end{array}$ & $\begin{array}{c}\text { O/p Image } \\
\text { in Kb }\end{array}$ \\
\hline 1 & 5.96 & 9.8 & 3.590361 & 3.769231 & 2.259231 \\
\hline 2 & 5.92 & 9.9 & 3.566265 & 3.807692 & 2.047692 \\
\hline 3 & 5.84 & 9.45 & 3.518072 & 3.634615 & 2.124615 \\
\hline 4 & 5.93 & 9.85 & 3.572289 & 3.788462 & 2.278462 \\
\hline 5 & 6.01 & 9.58 & 3.620482 & 3.684615 & 2.174615 \\
\hline 6 & 5.89 & 9.85 & 3.548193 & 3.788462 & 2.278462 \\
\hline 7 & 5.99 & 9.74 & 3.608434 & 3.746154 & 2.236154 \\
\hline 8 & 6.02 & 9.5 & 3.626506 & 3.653846 & 2.143846 \\
\hline 9 & 5.88 & 9.65 & 3.542169 & 3.711538 & 2.201538 \\
\hline 10 & 5.71 & 9.95 & 3.439759 & 3.826923 & 2.316923 \\
\hline & & & & & \\
\hline Average & 5.915 & 9.727 & 3.563253 & 3.741154 & 2.206154 \\
\hline
\end{tabular}

Table: 3 Stage by Stage average execution time

\begin{tabular}{|c|c|}
\hline Stage & $\begin{array}{c}\text { Time } \\
\text { taken (s) }\end{array}$ \\
\hline Preprocessing & 0.531 \\
\hline Feature Extraction & 3.494 \\
\hline Matrix Transpose \& addition & 0.016 \\
\hline Execution Time of proposed method & 11.587 \\
\hline
\end{tabular}

Table: 4 Performance of Proposed system

\begin{tabular}{|c|c|}
\hline PARAMETER & PROPOSED METHOD \\
\hline EXECUTION TIME & $11.587 \mathrm{~s}$ \\
\hline MEMORY ALLOCATION & $22.3 \mathrm{~KB}$ \\
\hline
\end{tabular}

\section{CONCLUSION}

In this paper, an advanced cancelable biometric framework utilizing Rotation Mobius transformation for multi-modular biometric has been planned and executed utilizing MTRMT system. It has been executed in MATLAB 2013.Here Iris and Fingerprint have been utilized as multimodal. Trial results are recorded and dissected. The results of the strategy has been as far as memory and Execution time. The Proposed framework utilizing MTRMT techniques shows better outcomes according to the ideal target.

\section{REFERENCES:}

1. A. A. Ross, K. Nandakumar, and A. K. Jain. Handbook of multibiometrics, volume 6. Springer, 2006.

2. H. Mehrotra, R. Singh, M. Vatsa, and B. Majhi, "Incremental granular relevance vectormachine: A case study in multimodal biometrics," Pattern Recognition, vol. 56, pp. 63-76, 2016.

3. M. Abdolahi, M. Mohamadi, and M. Jafari, "Multimodal biometric system fusion using fingerprint and iris with fuzzy logic," in International Journal of Soft Computing and Engineering, vol. 2, pp. 504-510, 2013.

4. D. Miao, M. Zhang, Z. Sun, T. Tan, and Z. He, "Bin-based classifier fusion of iris and face biometrics," Neurocomputing, vol. 224, pp. 105-118, 2017.

5. Y. Chen, J. Yang, C.Wang, andN. Liu, "Multimodal biometrics recognition based on local fusion visual features and variational Bayesian extreme learningmachine,"Expert Systems with Applications, vol. 64, pp. 93-103, 2016.

6. Kanade, S.; Petrovska-Delacrétaz, D.; Dorizzi, B. Cancelable iris biometrics and using error correcting codes to reduce variability in biometric data. In Proceedings of the IEEE Conference on Computer Vision and Pattern Recognition, Miami, FL, USA, 20-25 June 2009; pp. 120-127.

7. Pillai, J.K.; Patel, V.M.; Chellappa, R.; Ratha, N.K. Sectored random projections for cancelable iris biometrics. In Proceedings of the IEEE International Conference on Acoustics Speech and Signal Processing (ICASSP), Dallas, TX, USA, 14-19 March 2010; pp. 1838-1841.

8. K. Kanagalakshmi and E.Chandra, A Novel Technique for Cancelable and Irrevocable Biometric Template Generation for Fingerprints", Global Journal of Computer Science and Technology Graphics \& Vision, Vol. 13, Issue 6, pp.1-11, 2013.

9. K. Kanagalakshmi and E.Chandra, "Novel Complex Conjugate-Phase Transform technique for cancelable and irrevocable biometric template generation for fingerprints", IJCSI International Journal of Computer Science Issues, Vol. 9, Issue 4, No 2, pp: 426-436, July 2012.

10. Tristan Needham, Visual Complex Analysis, The Clarendon Press, Oxford University Press, New York, 1997.

\section{AUTHOR'S PROFILE}

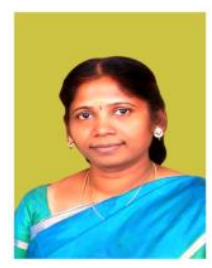

Dr. K. Kanagalakshmi, is working as Principal in Kamalam College of Arts and Science, Udumalpet,Tirupur Tamilnadu. She is having 19 years of teaching experience. She has published more than 16 research papers in International Journals and presented more than 66 papers in International, National and State level Seminars, Conferences together. Her areas of interest include Biometric, Pattern Recognition, Digital Image Processing, Security etc. She has produced more than 5 M.Phil. Scholars and she is guiding 8 Ph.D. Scholars. She has published 4 books. She is an Associate member of CSI. She received five awards like BEST FACULTY AWARD, BEST SCIENTIST AWARD etc. 


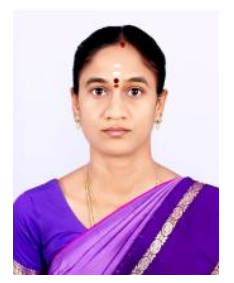

Mrs.K.Lakshmipriya, pursued M.Sc., M.Phil., under Bharathiar Univeristy. She is a part time doctorial research scholar in Nehru Arts and Science College, Coimbatore, Tamilnadu. She is also working as Head and Assistant Professor in department of computer science in Kamalam College of Arts and Science College,Udumalpet, Tirupur, Tamilnadu. She is having 10 years of teaching experience. She attended various national and international conferences and published research papers in various journals. Her area of interest is Data Mining and Biometrics. She received "GNANAGURU" award from Alagappa university study center \& Lion club of Pollachi. 